\title{
Early changes in quantitative ultrasound imaging parameters during neoadjuvant chemotherapy to predict recurrence in patients with locally advanced breast cancer
}

\section{Divya Bhardwaj}

Sunnybrook Health Sciences Centre

\section{Archya Dasgupta}

Sunnybrook Health Sciences Centre

Daniel DiCenzo

Sunnybrook Health Sciences Centre

Stephen Brade

Sunnybrook Health Sciences Centre

Kashuf Fatima

Sunnybrook Health Sciences Centre

Karina Quiaoit

Sunnybrook Health Sciences Centre

Maureen Trudeau

Sunnybrook Health Sciences Centre

Sonal Gandhi

Sunnybrook Health Sciences Centre

Andrea Eisen

Sunnybrook Health Sciences Centre

Frances Wright

Sunnybrook Health Sciences Centre

Nicole Look-Hong

Sunnybrook Health Sciences Centre

Belinda Curpen

Sunnybrook Health Sciences Centre

Lakshmanan Sannachi

Sunnybrook Health Sciences Centre

Gregory Czarnota ( $\nabla$ gregoryczarnota.submissions@gmail.com )

Sunnybrook Health Sciences Centre https://orcid.org/0000-0002-0519-2182 


\section{Research Article}

Keywords: Radiomics, Breast cancer, Recurrence, Quantitative ultrasound, Neoadjuvant chemotherapy, Delta Radiomics, Machine learning, Texture analysis, Texture Derivatives, Imaging biomarker.

Posted Date: June 4th, 2021

DOI: https://doi.org/10.21203/rs.3.rs-566597/v1

License: (c) (i) This work is licensed under a Creative Commons Attribution 4.0 International License. Read Full License

Version of Record: A version of this preprint was published at Cancers on February 28th, 2022. See the published version at https://doi.org/10.3390/cancers14051247. 


\section{Abstract}

Background: This study was conducted in order to explore the use of quantitative ultrasound (QUS) based higher-order texture derivatives in predicting recurrence for patients with locally advanced breast cancer (LABC) early during neoadjuvant chemotherapy (NAC).

Methods: Eighty-three patients with LABC were scanned with $7 \mathrm{MHz}$ ultrasound before starting NAC (week 0 ) and during treatment (week 4). Spectral parametric maps were generated corresponding to tumor volume. Twenty-four textural features (QUS-Tex ${ }^{1}$ ) were determined from parametric maps acquired using grey-level co-occurrence matrices (GLCM) for each patient, which were further processed to generate 64 texture derivatives (QUS-Tex ${ }^{1}-\mathrm{Tex}^{2}$ ), leading to a total of 95 features from each time points. Analysis was carried out on week 4 data and compared to baseline (week 0 ) data. $\Delta$ Week 4 data was obtained from the difference in QUS parameters, texture features (QUS-Tex ${ }^{1}$ ), and texture derivatives (QUS-Tex ${ }^{1}-$ Tex $^{2}$ ) of week 4 data and week 0 data. Patients were divided into two groups based on their clinical status: recurrence and no-recurrence. Machine learning algorithms using $k$-nearest neighbor (k-NN) and support vector machines (SVM) were used to generate radiomic models.

Results: With a median follow up of 69 months (range 7-118 months), 28 patients had disease recurrence. The k-NN classifier was the best performing algorithm at week 4 with sensitivity, specificity, accuracy, and area under curve(AUC) of $87 \%, 75 \%, 81 \%$, and 0.83 , respectively. The inclusion of texture derivatives (QUS-Tex ${ }^{1}-\mathrm{Tex}^{2}$ ) in week 4 QUS data analysis led to the improvement of the classifier performances. The AUC increased significantly from 0.70 (without texture derivatives) to 0.83 (with texture derivatives). The most relevant features in separating the two groups were higher-order texture derivatives obtained from scatterer diameter and acoustic concentration-related parametric images.

Conclusion: This is the first study highlighting the utility of QUS radiomics in the prediction of recurrence during the treatment of $L A B C$. It reflects that the ongoing treatment-related changes can predict clinical outcomes with higher accuracy as compared to pretreatment features alone.

Clinical Trial Registration:ClinicalTrials.gov Identifier: NCT00437879.

Registry name: Clinicaltrials.gov.

Date of registration: $20^{\text {th }}$ February 2007

URL: https://clinicaltrials.gov/ct2/show/study/NCT00437879

\section{Introduction}

Breast cancer is the most commonly diagnosed malignancy and is the leading cause of cancer-related death among women.[1] Locally advanced breast cancer (LABC) can be clinically defined as a primary tumor larger than $5 \mathrm{~cm}$ in size, which may be fixed to the chest wall, with advanced lymph node 
involvement. (LABC) poses a clinical challenge because, despite aggressive multimodality treatment, these patients have a high rate of recurrence.[2,3] Neoadjuvant chemotherapy (NAC) is often used in patients with $L A B C$ with the purpose of downstaging disease, improving resectability, and also treatment response can serve as a useful indicator of long-term survival outcomes in specific molecular subgroups[4]. Approximately $30-50 \%$ of patients with LABC after primary treatment present with recurrence, which is influenced by prognostic factors as well as treatment strategies.[2] For breast cancer patients, it has been demonstrated that the fear of cancer recurrence to be one of the most common and aversive psychological phenomena.[5] Genomic tools have been developed in estimating recurrence risk in patients with early-stage breast cancer and incorporated into clinical practice, guiding decisions regarding adjuvant therapy.[6-8] However, most of these genomic assays have limited clinical utility in $\angle A B C$ so far. In regards to imaging tools, limited literature exists on the use of imaging biomarkers in predicting the risk of breast cancer recurrence. In the past decade, a growing interest in the field of radiomics using quantitative imaging analysis has led to significant advancements in image-based phenotyping aimed at tumor characterization, and recently the identification of predictors of treatment response in patients with breast cancer.[9] Radiomics reflects the mechanisms occurring at a genetic and molecular level by converting imaging data into high dimensional, mineable quantitative features.

Ultrasound (US) is a commonly used imaging modality with widespread clinical applications in differentiating benign versus malignant primary lesions in the breast or characterizing lymph nodes and facilitating tissue acquisition for histological evaluation. Quantitative ultrasound (QUS) is similar to the clinically used ultrasound device, with the primary difference of acquisition and analyzing the raw radiofrequency (RF) data which aids in more detailed biological characterization of the imaged tissues. The biophysical basis of QUS imaging is the differences in the elastic properties, leading to differential scattering reflected as backscatter ultrasound signals from tissues[10,11]. Application of quantitative texture analysis to the QUS imaging has shown to unfold critical biological information that can be modeled effectively to predict biological behavior in breast malignancies $[12,13]$. Several studies have demonstrated the utility of QUS imaging in predicting treatment response using pretreatment imaging features $[14,12,15,16]$. Similarly, the acquisition of QUS features during different treatment modalities (chemotherapy, radiotherapy) reflects ongoing microstructural changes resulting from cell death and can effectively improve the interpretation of clinical outcomes $[17,18]$.

The study here explored the role of QUS features, including higher-order texture features obtained during the early course of NAC, in predicting the recurrence for patients with $L A B C$.

\section{Material And Methods}

\section{Patient Selection}

The prospective study was approved by the Research Ethics Board at Sunnybrook Health Sciences Centre (SHSC), Toronto, Canada, and registered with Clinical Trials.gov (NCT00437879). All study participants signed a written consent form. Inclusion criteria were the presence of biopsy-proven LABC and scheduled 
to receive NAC as part of their standard care of treatment. Clinical information was obtained from a prospectively maintained database with additional information collected from patient electronic medical records. Patients diagnosed with inflammatory breast cancer, upfront metastasis, or having a second were excluded from the analysis. The time to recurrence was defined from the start of NAC to the clinical or radiological evidence of disease recurrence. In order to avoid underestimating the risk of recurrence, the minimum follow-up time for patients without any recurrence was required to be at least four years.

\section{Treatment Details}

The majority of patients were treated with an anthracycline and taxane-based NAC, while some patients received other chemotherapy treatments. The chemotherapy regimen and the interval between two cycles were based on the decision of the treating medical oncologist. Patients with human epidermal growth factor receptor 2 (HER2) positive status received targeted therapy in combination with NAC. After receiving NAC, all patients underwent surgery (mastectomy or breast-conserving surgery), in conjunction with sentinel lymph node biopsy (SLNB) or axillary lymph node dissection (ALND), based on the pretreatment disease stage and response. Patients received adjuvant locoregional radiation therapy and maintenance targeted therapy or endocrinal therapy according to standard institutional practice. Patients were followed up every 3-6 months during the first two years, and then after every 6-12 months, as indicated clinically. Oncologists confirmed all recurrences with clinical examination, diagnostic imaging, and histological confirmation as indicated clinically.

\section{Quantitative Ultrasound Parameter Estimation}

Ultrasound data were obtained using a Sonix RP clinical research system (Analogic Medical Corp., Vancouver, Canada) coupled to a L14-5/60 transducer with a central frequency of $7 \mathrm{MHz}$ and $-6 \mathrm{~dB}$ bandwidth of 4-9 MHz. Data were digitally collected with a sampling frequency of $40 \mathrm{MHz}$ with a 16-bit resolution. Patients were scanned before the initiation of systemic therapy (week 0 ) and at week 4 from the start date of NAC. The scan frames were collected at intervals of $1 \mathrm{~cm}$, spanning the entire tumor, with the transducer focus towards the center of the tumor. On each ultrasound frame, a region of interest (ROI) was manually contoured corresponding to the tumor. Using a sliding window approach, each ROI was divided into analysis blocks of size $10 \lambda \times 10 \lambda$, with a $94 \%$ adjacent overlap in axial and lateral directions (2.2 $\mathrm{mm} \times 2.2 \mathrm{~mm}$ approximately). The detailed description of standardization methods and calculation of quantitative ultrasound parameters have been explained in previous publications.[19] In short, from each of the selected ultrasound frames, spectral parameters including mid-band fit (MBF), spectral slope (SS), spectral intercept (SI), spacing among scatterers (SAS) and backscatter model parameters, acoustic scatterer diameter- Anderson (ASD) and average acoustic-scatterer concentration (AAC) were determined. [20][21] Attenuation coefficient estimation (ACE) was used as a spectral correction factor and also as a predictive parameter.[22][23] Spectral parametric maps, represented as color-coded image maps, were generated for each ROls through the spatial mapping of the parametric values calculated from all window blocks. Mean QUS parameters were calculated by averaging the values within the maps.

\section{Texture Features and Texture Derivatives- Evaluation:}


The spatial distribution of QUS parameters was evaluated from parametric maps using grey-level cooccurrence matrices (GLCM)[24]' representing the distance and angular spatial relationship between neighboring pixels]. From each parametric map, the following four textural features were extracted; contrast parameter $(\mathrm{CON})$, correlation parameter (COR), homogeneity parameter ( $\mathrm{HOM})$, and energy parameter (ENE).

In summary, the CON parameter measures the magnitude of intensity differences between two neighboring pixels. The COR parameter is a measure of how linearly a pixel is correlated to its adjacent pixel. The ENE parameter measures textural uniformity, and the HOM parameter estimates the incidence of pixel pairs of different intensities. A total of 24 textural features (QUS-Tex ${ }^{1}$ ) were determined from the 6 spectral parametric maps (excluding ACE).

In order to determine third-order imaging features (texture derivatives), the GLCM method (ENE, CON, COR, HOM) was repeated on the 16 QUS-texture maps (excluding SAS and SS textural features) described above. As a result, a total of 64 texture derivatives were determined (QUS-Tex ${ }^{1}-T^{-}{ }^{2}{ }^{2}$ ). The steps were repeated for all ultrasound scans obtained (at weeks 0 and 4). The $\Delta$ week 4 data was obtained from the difference in mean QUS parameters, texture features (QUS-Tex ${ }^{1}$ ), and texture derivatives (QUS-Tex ${ }^{1}-$ Tex $^{2}$ ) of week 4 data from the corresponding week 0 data.

Therefore we had a set of 95 features from each time point (7 QUS spectral features, 24 QUS-Tex ${ }^{1}$, and 64 QUS Tex ${ }^{1}-T^{2}{ }^{2}$ ). Final analysis was carried out using a combination of week 0 and $\Delta$ week 4 data (190 features).

\section{Statistical Analysis and Classification Algorithms}

A Shapiro-Wilk normality test was applied to the feature sets to confirm data distribution for the two groups (patients with and without recurrence). For normally distributed faculty, a parametric unpaired student t-test was used, and otherwise, a non-parametric test Mann-Whitney $U$ test was performed. A total of 190 features (7 mean QUS, 24 QUS-Tex ${ }^{1}, 64$ QUS-Tex ${ }^{1}-$ Tex $^{2}$ from each point), which was a combination of week 0 data and $\Delta$ Week 4 data, were used for the model development. A p-value of < 0.05 was considered statistically significant.

Two machine learning classifiers, consisting of k-nearest neighbor (k-NN), and support vector machineradial based function (SVM), were independently tested for comparison using MATLAB 2016a to evaluate diagnostic performance. The maximum feature number was set to 3 in the classification model to avoid model overfitting due to the high dimensionality of the data set. [25] Since the two groups were not balanced well ( 28 with recurrence and 55 without recurrence), subset sampling method was undertaken. Seven subsets were generated randomly generated to select an equal number of patients equivalent to the smaller group (recurrence), with the final class assignment done based on majority voting. A forward feature selection method was used for building the classifier algorithms. A leave-one patient out crossvalidation method was used, which uses all except one data point in the prediction of the output class. The performances of the different algorithms were compared based on different diagnostic indices 
obtained, including sensitivity, specificity, accuracy, and area under curve (AUC). To test the incremental values of higher-order features (texture derivatives), the classifier models were tested separately including QUS and QUS-Tex1 features, and then with all the features combined.

Survival analysis was undertaken using Kaplan Meier product-limit method. Univariate analysis was done using log-rank tests to compare the survival estimates for the two predicted groups using the radiomics model (predicted recurrence group versus predicted non-recurrence group).

\section{Results}

\section{Clinical Characteristics:}

Analysis included a sample size of 83 women with $L A B C$ as per inclusion criteria. The patient's ages ranged from 29 to 79 years (median 50 years). Clinical characteristics, histopathologic features, and treatment details have been mentioned in Table 1. To summarize, the histology was invasive ductal carcinoma (IDC) in 76, invasive lobular carcinoma (ILC) in 3, mixel IDC and ILC in 3, and metaplastic carcinoma in 1. Fifty-six patients received doxorubicin, cyclophosphamide, and paclitaxel (AC-T), and twenty patients were administered fluorouracil, epirubicin, cyclophosphamide, and docetaxel (FEC-D). Twenty-nine patients were HER2 positive and, forty-eight were estrogen receptor (ER) positive.

\section{Quantitative Ultrasound Feature Analysis:}

Representative ultrasound B-mode images and parametric maps of QUS features and selected texture and texture-derivative maps for a patient with (1a) and without recurrence (1b) are presented in Fig. 1. $\triangle$ ASD-ENE $(p=0.033)$, and $\triangle$ MBF-HOM-CON $(p=0.038)$ parameters exhibited a statistically significant difference between the two groups of patients (Table 2). The corresponding scatter plots for these two features as obtained from all the patients are shown in Fig. 2. Representative data for all QUS, texture and texture-derivative features are shown in Supplementary Fig. 1.

\section{Classifier Results:}

Results obtained using different classifier models are displayed in Table 3, and their associated receiver operating characteristic (ROC) curves and AUC values are presented in Fig. 3. The performance of both classifier models, k-NN and SVM based on data acquired at a week 4 time, had an accuracy above $70 \%$. Specifically, it was observed that using the k-NN classifier model, the week 4 data in aggregate (QUS + QUS-Tex ${ }^{1}+$ QUS-Tex ${ }^{1}-$ Tex $^{2}$ ), performed better as compared to the week 0 data (QUS + QUS-Tex ${ }^{1}+$ QUSTex ${ }^{1}-\mathrm{Tex}^{2}$ ). The AUC improved from 0.78 to 0.83 in the k-NN classifier model, whereas it only enhanced marginally in the SVM model, from 0.76 to 0.78 (Figs. 3a \& 3b).

At week 4 , there was a significant improvement observed in the performance of the k-NN classifier model with the introduction of texture derivatives (QUS-Tex ${ }^{1}-\mathrm{Tex}^{2}$ ). The AUC improved significantly from 0.70 to 0.83 (Fig. 3c). For the SVM model, the AUC of 0.78 remained the same, with no difference seen with the incorporation of texture derivatives. (Fig. 3d). 
Overall, the k-NN classifier performed best at week 4; the selected best features were a multiparametric combination of $\mathrm{ACE}_{\mathrm{W} 0}, \mathrm{AAC}-\mathrm{CON}-\mathrm{CON}_{\mathrm{W}}$, and $\triangle \mathrm{ASD}-\mathrm{CON}-\mathrm{CON}$. The sensitivity, specificity, and accuracy at week 4 for this model were $87 \%, 75 \%$, and $81 \%$, respectively.

\section{Clinical Outcomes and Performance of Prediction Models:}

The median follow up for the entire cohort was 64 months (interquartile range 49-84 months). Out of eighty-three patients included in the analysis, 28 patients had developed disease recurrence, with the median time to recurrence being 24 months. More than $80 \%$ of the recurrences were seen in the first four years. The site of the first recurrence was isolated local disease in 5, local and regional in 1 , regional and distant in 4, isolated distant relapse in 17, and combined local, regional, and distant in 1 patient. For the entire group, 5-year recurrence-free survival (RFS) and 5-year overall survival (OS) were 68\% and 79\%, respectively. The predicted RFS using the k-NN and SVM classifier has been shown in Fig. 4. The 5-year RFS for the predicted recurrence and non-recurrence groups using the k-NN classifier was 56\% and 93\%, respectively $(p=0.001)$.

\section{Discussion}

Several studies have identified various factors that can predict the recurrence of breast cancer ranging from clinical characteristics, immunohistochemical assays to gene expression levels. Clinically, the most reliable prognostic markers include nodal status and tumor size. Various other clinical factors such as tumor grade, patient age, and treatment type have been added to build a Clinical Treatment Score (CTS), which provides a recurrence risk estimate for ER + breast cancer.[26] In the past two decades, there has been a range of genetic markers to predict the risk of cancer recurrence, primarily for $\mathrm{EBC} .[6,27,28]$. Some studies have compared the genetic analysis with imaging features showing good correlations. In a study by Sutton et al. (2015), the association between the gene assay recurrence score and texture-based image features extracted from magnetic resonance imaging (MRI) was investigated.[29]. Similarly, a study by Woodard et al. (2018) showed equivalent efficacy of the gene assay and Breast Imaging and Reporting Data System (BI-RADS) mammography and MR images in the prediction of recurrence. [30]

The role of ultrasound is widely recognized in the screening and diagnosis of breast masses. Applications include assessing morphological tumor details (spiculated, rounded, with necrosis, microcalcification), anatomic relationships of masses to their surrounding tissues, and regional lymph nodal involvement. However, recent clinical applications of ultrasound have expanded in the management of breast cancer. For example, ultrasound elastography techniques can be used to differentiate benign from malignant breast lesions, contrast-enhanced ultrasound can characterize tissues with different vascularity, and three-dimensional ultrasound improves the characterization of breast lesions.[31] QUS Techniques have been applied in characterizing tumor masses and predicting treatment response in patients with $\mathrm{LABC} .[17,17,32]$

In the past, it has been demonstrated in preclinical studies that QUS can evaluate cell death in response to different treatment modalities.[33,34] The intracellular mechanisms resulting in phenotypical cell 
changes differ according to the mode of cell death. For example, mitotic arrest results in cell swelling, whereas apoptotic death leads to cell shrinkage, chromatin condensation, and nuclear fragmentation. These events form the basis for differential change in QUS spectral and textural parameters for cell death monitoring. These observations have been interpreted in clinical studies to predict treatment response within 4 weeks of initiation of NAC.[35]

The work presented in this study aimed to investigate the effectiveness of QUS texture derivatives in predicting recurrence risk rather than the local response in breast cancer within weeks of initiation of systemic therapy. The improvement in AUC performance with the addition of texture derivatives, as well as from week 0 to week 4 in the k-NN classifier, signifies the importance of higher-order derivatives and continuous monitoring. It was interesting to observe that the best features chosen by the k-NN model to classify patients in the two groups mainly were texture derivatives (texture of texture features). The features were a combination of ACE $_{\mathrm{W} 0}, \mathrm{AAC}-\mathrm{CON}^{-} \mathrm{CON}_{\mathrm{W}}$, and $\triangle \mathrm{ASD}-\mathrm{CON}-\mathrm{CON}$, at week 4 (Week $0+$ $\triangle$ Week 4). Amongst the three features, the two parameters $\left(A C E_{W_{0}} \& A A C-C O N-C O N_{W 0}\right.$ ) are related to tissue composition and microstructural organization of cells. The selection of texture derivative 'AAC$\mathrm{CON}-\mathrm{CON}_{\mathrm{WO}}$ ' technically represents the summation of other sub-variables and possibly depicts heterogeneity within a tumor at a more advanced level. Other parameters were selected from the pretreatment (week 0 ) data. This suggests that the spatial organization of the tissue as reflected in QUS parameters relates to tumor biology and may have a role in recurrence prediction to some extent before initiation of treatment. In previous studies, $\mathrm{ACE}_{\text {wo }}$ was found to be a significant predictive parameter to distinguish tissues of different types, supporting the findings in this study.[36, 37]

The other texture derivative selected was $\triangle$ ASD-CON-CON, where ASD signifies microstructural size and may be related to lobular diameter, and the contrast derivatives (contrast of contrast), which measure the intensity differences. This was found to be significant after the initiation of treatment. As mentioned previously, changes occurring at the molecular level in tumor cells in response to the treatment can be captured by the QUS technique. Hence, it is postulated that as treatment initiates, ASD and its derivatives have a potential role in predicting recurrence risk and differentiating the two groups. -free survival.

We estimated the survival outcomes based on a prediction by the two machine learning classifiers. Out of the two, the k-NN classifier using texture derivatives (week 4) was able to closely approximate the curve obtained from the patient's clinical details on recurrence, thus displaying the efficacy of the classifier in recurrence prediction.

One of the limitations of the study here was the relatively small number of patients who presented with recurrence. The inclusion of more patients will likely improve the performance and robustness of the classifiers. In the future, with a larger cohort, it may also be worthwhile to combine clinical features and molecular subgroups with a QUS-radiomics model. The promising results obtained in the study here emphasize the importance of QUS parameters as a valuable tool for the timely identification of patients whose tumors have a strong tendency towards recurrence. An early prediction of recurrence risk can potentially assist oncologists to make decisions in regards to selecting systemic agents for treatment or 
changing a less effective treatment to more effective therapy or maintenance therapies. It may provide an insight into an earlier shift to surgery or towards an intensification of systemic therapy before missing the "therapeutic window" for benefit.

\section{Conclusion}

To summarize, this study demonstrates the effectiveness of QUS-radiomics in predicting recurrence in patients with $L A B C$ early in the course of treatment. The work presented here highlights the role of higherorder imaging features (texture-derivatives) in improving the performance of the classifiers at 4 weeks from into neoadjuvant chemotherapy.

\section{Declarations}

Ethics approval and consent to participate: The study was approved by the Research Ethics Board of Sunnybrook Health Sciences Centre. Written concept was obtained from all the study patients.

Consent for publication: Not required.

Competing interests: Nothing to declare

\section{Funding:}

1. Terry Fox Foundation Program Project Grant from the Hecht Foundation (grant number 1083)

2. Canadian Institutes of Health Research (CIHR) (Grant number PJT 159759)

The funding agencies had no role in the study concept, design, analysis, interpretation of data, and manuscript preparation.

\section{Author contributions:}

Conceptualization: AD, GJC; Methodology: All authors; Formal Analysis and investigation: All authors; Writing-original draft preparation: DB, AD, GJC; Writing-review and editing: All authors; Project administration and supervision: GJC; Funding acquisition: GJC

All the authors are in agreement and accountable for all the aspects of the work.

\section{Availability of supporting data}

Anonymized data will be provided on request to the corresponding author according to institutional ethics committee guidelines.

Acknowledgments: We would like to thank all the patients for their participation in the study and their caregivers for continuous support. Our sincere gratitude to the Terry Fox Foundation and the Canadian 
Institutes of Health Research for funding support. We express our regard to the physicians and other health care staff for their support in patient care.

\section{References}

1. Bray F, Ferlay J, Soerjomataram I, Siegel RL, Torre LA, Jemal A. Global cancer statistics 2018: GLOBOCAN estimates of incidence and mortality worldwide for 36 cancers in 185 countries. CA: A Cancer Journal for Clinicians. 2018;68:394-424.

2. Tryfonidis K, Senkus E, Cardoso MJ, Cardoso F. Management of locally advanced breast cancerperspectives and future directions. Nat Rev Clin Oncol. 2015;12:147-62.

3. Wang M, Hou L, Chen M, Zhou Y, Liang Y, Wang S, et al. Neoadjuvant Chemotherapy Creates Surgery Opportunities For Inoperable Locally Advanced Breast Cancer. Sci Rep. 2017;7:44673.

4. Vaidya JS, Massarut S, Vaidya HJ, Alexander EC, Richards T, Caris JA, et al. Rethinking neoadjuvant chemotherapy for breast cancer. BMJ. 2018;360:j5913.

5. Ziner KW, Sledge GW, Bell CJ, Johns S, Miller KD, Champion VL. Predicting fear of breast cancer recurrence and self-efficacy in survivors by age at diagnosis. Oncology Nursing Forum. NIH Public Access; 2012;39:287-95.

6. Van't Veer LJ, Dai H, Van de Vijver MJ, He YD, Hart AAM, Mao M, et al. Gene expression profiling predicts clinical outcome of breast cancer. Nature. Nature Publishing Group; 2002;415:530-6.

7. Sparano JA, Gray RJ, Makower DF, Pritchard KI, Albain KS, Hayes DF, et al. Adjuvant Chemotherapy Guided by a 21-Gene Expression Assay in Breast Cancer. N Engl J Med. 2018;379:111-21.

8. Sparano JA, Gray RJ, Makower DF, Albain KS, Saphner TJ, Badve SS, et al. Clinical Outcomes in Early Breast Cancer With a High 21-Gene Recurrence Score of 26 to 100 Assigned to Adjuvant Chemotherapy Plus Endocrine Therapy: A Secondary Analysis of the TAILORx Randomized Clinical Trial. JAMA Oncol. 2020;6:367-74.

9. Tagliafico AS, Piana M, Schenone D, Lai R, Massone AM, Houssami N. Overview of radiomics in breast cancer diagnosis and prognostication. Breast. 2020;49:74-80.

10. Czarnota GJ, Kolios MC, Abraham J, Portnoy M, Ottensmeyer FP, Hunt JW, et al. Ultrasound imaging of apoptosis: high-resolution non-invasive monitoring of programmed cell death in vitro, in situ and in vivo. British Journal of Cancer. Nature Publishing Group; 1999;81:520-7.

11. Feleppa EJ, Mamou J, Porter CR, Machi J. Quantitative ultrasound in cancer imaging. Semin Oncol. 2011;38:136-50.

12. Dasgupta A, Brade S, Sannachi L, Quiaoit K, Fatima K, DiCenzo D, et al. Quantitative ultrasound radiomics using texture derivatives in prediction of treatment response to neo-adjuvant chemotherapy for locally advanced breast cancer. Oncotarget. 2020;11:3782-92.

13. Osapoetra LO, Sannachi L, Quiaoit K, Dasgupta A, DiCenzo D, Fatima K, et al. A priori prediction of response in multicentre locally advanced breast cancer ( $L A B C)$ patients using quantitative ultrasound and derivative texture methods. Oncotarget. 2021;12:81-94. 
14. DiCenzo D, Quiaoit K, Fatima K, Bhardwaj D, Sannachi L, Gangeh M, et al. Quantitative ultrasound radiomics in predicting response to neoadjuvant chemotherapy in patients with locally advanced breast cancer: Results from multi-institutional study. Cancer Med. 2020;

15. Dasgupta A, Fatima K, DiCenzo D, Bhardwaj D, Quiaoit K, Saifuddin M, et al. Quantitative ultrasound radiomics in predicting recurrence for patients with node-positive head-neck squamous cell carcinoma treated with radical radiotherapy. Cancer Med. 2020;

16. Osapoetra LO, Dasgupta A, DiCenzo D, Fatima K, Quiaoit K, Saifuddin M, et al. Assessment of clinical radiosensitivity in patients with head-neck squamous cell carcinoma from pre-treatment quantitative ultrasound radiomics. Sci Rep. 2021;11:6117.

17. Quiaoit K, DiCenzo D, Fatima K, Bhardwaj D, Sannachi L, Gangeh M, et al. Quantitative ultrasound radiomics for therapy response monitoring in patients with locally advanced breast cancer: Multiinstitutional study results. PLOS ONE. Public Library of Science; 2020;15:e0236182.

18. Fatima K, Dasgupta A, DiCenzo D, Kolios C, Quiaoit K, Saifuddin M, et al. Ultrasound delta-radiomics during radiotherapy to predict recurrence in patients with head and neck squamous cell carcinoma. Clin Transl Radiat Oncol. 2021;28:62-70.

19. Tadayyon H, Sadeghi-Naini A, Czarnota GJ. Noninvasive characterization of locally advanced breast cancer using textural analysis of quantitative ultrasound parametric images. Translational Oncology. Elsevier B.V.; 2014;7:759-67.

20. Lizzi FL, Feleppa EJ, Kaisar Alam S, Deng CX. Ultrasonic spectrum analysis for tissue evaluation. Pattern Recognition Letters. 2003;24:637-58.

21. Insana MF, Hall TJ. Coefficient Measurements: Image Formation and. 2015;267:245-67.

22. Labyed $Y$, Bigelow TA. A theoretical comparison of attenuation measurement techniques from backscattered ultrasound echoes. The Journal of the Acoustical Society of America. 2011;129:2316-24.

23. Tro IN, Tion DUC. Ap pli ca tion of Three Scat ter ing Mod els to Char ac ter iza tion of Solid Tu mors in Mice. 2015;96:83-96.

24. Haralick RM, Shanmugam K, Dinstein I. Textural Features for Image Classification. IEEE Transactions on Systems, Man, and Cybernetics. 1973;SMC-3:610-21.

25. Jain AK, Duin RPW, Mao J. Statistical pattern recognition: A review. IEEE Transactions on Pattern Analysis and Machine Intelligence. IEEE; 2000;22:4-37.

26. Dowsett M, Sestak I, Regan MM, Dodson A, Viale G, Thurlimann B, et al. Integration of clinical variables for the prediction of late distant recurrence in patients with estrogen receptor-positive breast cancer treated with 5 years of endocrine therapy: CTS5. Journal of Clinical Oncology. 2018;36:1941-8.

27. Paik S, Shak S, Tang G, Kim C, Baker J, Cronin M, et al. A multigene assay to predict recurrence of tamoxifen-treated, node-negative breast cancer. New England Journal of Medicine. 2004;351:281726. 
28. Prat A, Parker JS, Fan C, Perou CM. PAM50 assay and the three-gene model for identifying the major and clinically relevant molecular subtypes of breast cancer. Breast Cancer Research and Treatment. 2012;135:301-6.

29. Sutton EJ, Oh JH, Dashevsky BZ, Veeraraghavan H, Apte AP, Thakur SB, et al. Breast cancer subtype intertumor heterogeneity: MRI-based features predict results of a genomic assay. Journal of Magnetic Resonance Imaging. John Wiley and Sons Inc.; 2015;42:1398-406.

30. Woodard GA, Ray KM, Joe BN, Price ER. Qualitative radiogenomics: Association between oncotype DX test recurrence score and BI-RADS mammographic and breast MR imaging features. Radiology. 2018;286:60-70.

31. Guo R, Lu G, Qin B, Fei B. Ultrasound Imaging Technologies for Breast Cancer Detection and Management: A Review. Ultrasound in Medicine and Biology. Elsevier USA; 2018. p. 37-70.

32. Sadeghi-Naini A, Sannachi L, Pritchard K, Trudeau M, Gandhi S, Wright FC, et al. Early prediction of therapy responses and outcomes in breast cancer patients using quantitative ultrasound spectral texture. Oncotarget. 2014;5:3497-511.

33. Pasternak MM, Wirtzfeld LA, Kolios MC, Czarnota GJ. High-frequency ultrasound analysis of postmitotic arrest cell death. Oncoscience. 2016;3:109-21.

34. Pasternak MM, Sadeghi-Naini A, Ranieri SM, Giles A, Oelze ML, Kolios MC, et al. High-frequency ultrasound detection of cell death: Spectral differentiation of different forms of cell death in vitro. Oncoscience. 2016;3:275-87.

35. Sannachi L, Gangeh M, Tadayyon H, Gandhi S, Wright FC, Slodkowska E, et al. Breast Cancer Treatment Response Monitoring Using Quantitative Ultrasound and Texture Analysis: Comparative Analysis of Analytical Models. Translational Oncology. Elsevier Ltd; 2019;12:1271-81.

36. D'Astous FT, Foster FS. Frequency dependence of ultrasound attenuation and backscatter in breast tissue. Ultrasound in Medicine and Biology. Elsevier; 1986;12:795-808.

37. Landini L, Sarnelli R. Evaluation of the attenuation coefficients in normal and pathological breast tissue. Medical \& biological engineering \& computing. 1986;24:243-7.

\section{Tables}

Table 1: Patient, disease, and treatment-related characteristics for all patients $(n=83)$. 


\begin{tabular}{|c|c|c|c|c|c|}
\hline \multirow{2}{*}{\multicolumn{2}{|c|}{$\begin{array}{ll}\text { Features } \\
\text { Patient Characteristics }\end{array}$}} & \multicolumn{2}{|c|}{ Recurrence $(\mathrm{n}=28)$} & \multicolumn{2}{|c|}{ Non-recurrence $(n=55)$} \\
\hline & & $\mathbf{n}$ & $\%$ & $\mathbf{n}$ & $\%$ \\
\hline Age & Median (Range) & \multicolumn{2}{|c|}{$50(29-79)$ years } & \multicolumn{2}{|c|}{$48(31-72)$ years } \\
\hline \multirow[b]{4}{*}{ Menopausal Status } & Premenopausal & 16 & 57 & 33 & 60 \\
\hline & Perimenopausal & 1 & 4 & 3 & 6 \\
\hline & Postmenopausal & 10 & 36 & 17 & 31 \\
\hline & Not specified & 1 & 4 & 2 & 4 \\
\hline \multirow[t]{2}{*}{ Laterality } & Right & 15 & 54 & 27 & 49 \\
\hline & Left & 13 & 46 & 28 & 51 \\
\hline \multicolumn{2}{|l|}{ Pathological features } & $\mathbf{n}$ & $\%$ & $\mathbf{n}$ & $\%$ \\
\hline \multirow[t]{3}{*}{ Histology } & IDC & 25 & 89 & 51 & 93 \\
\hline & ILC & 2 & 7 & 1 & 2 \\
\hline & Others & 1 & 4 & 3 & 5 \\
\hline \multirow[t]{2}{*}{ ER Status } & Negative & 13 & 46 & 22 & 40 \\
\hline & Positive & 15 & 54 & 33 & 60 \\
\hline \multirow[t]{2}{*}{ PR Status } & Negative & 13 & 46 & 27 & 49 \\
\hline & Positive & 15 & 54 & 28 & 51 \\
\hline \multirow[t]{2}{*}{ HER2 Status } & Negative & 18 & 64 & 36 & 66 \\
\hline & Positive & 10 & 36 & 19 & 34 \\
\hline \multicolumn{2}{|l|}{ Neoadjuvant Treatment } & $\mathbf{n}$ & $\%$ & $\mathbf{n}$ & $\%$ \\
\hline \multirow[t]{3}{*}{ Chemotherapy regimen } & AC-T & 21 & 75 & 35 & 64 \\
\hline & FEC-D & 5 & 18 & 15 & 27 \\
\hline & TC & 2 & 7 & 5 & 8 \\
\hline \multirow[t]{2}{*}{ Dose Dense } & No & 13 & 46 & 26 & 47 \\
\hline & Yes & 15 & 54 & 29 & 53 \\
\hline \multirow[b]{2}{*}{ Trastuzumab } & No & 18 & 64 & 36 & 66 \\
\hline & Yes & 10 & 36 & 19 & 34 \\
\hline \multicolumn{2}{|l|}{ Treatment Response } & $\mathbf{n}$ & $\%$ & $\mathbf{n}$ & $\%$ \\
\hline \multicolumn{2}{|c|}{ Pathological Complete Response (pCR) } & 0 & 0 & 16 & 29 \\
\hline \multicolumn{2}{|c|}{ Partial Responder (PR) } & 21 & 75 & 33 & 60 \\
\hline \multicolumn{2}{|l|}{ Non Responder (NR) } & 7 & 25 & 6 & 11 \\
\hline
\end{tabular}

Abbreviations: ER: Estrogen receptor; PR: Progesterone receptor; HER2+: Human epidermal growth factor receptor 2; IDC: Invasive ductal carcinoma; ILC: Invasive lobular carcinoma; AC-T: doxorubicin, cyclophosphamide, and docetaxel, FEC-D: 5-fluorouracil, epirubicin, cyclophosphamide, and docetaxel; TC: docetaxel and cyclophosphamide

Table 2: Features with significant differences at week four into neoadjuvant chemotherapy.

\begin{tabular}{|l|c|c|c|}
\hline \multicolumn{1}{|c|}{ Parameter } & $\begin{array}{c}\text { Recurrence } \\
\text { Mean } \pm \text { SEM }\end{array}$ & $\begin{array}{c}\text { Non-recurrence } \\
\text { Mean } \pm \text { SEM }\end{array}$ & -value \\
\hline$\Delta$ ASD-ENE & $0.008 \pm 0.021$ & $0.005 \pm 0.099$ & 0.033 \\
\hline$\Delta$ MBF-HOM-CON & $-0.306 \pm 0.889$ & $0.160 \pm 0.867$ & 0.038 \\
\hline
\end{tabular}


$* \Delta$ Indicates the difference of values of week 4 from week 0 for each feature included in the analysis

Abbreviations: SEM: standard error of the mean; R: Recurrence; NR: No Recurrence; ASD: Average Scatterer Diameter; MBF: Mid-band fit; AAC: Average Acoustic Concentration; ENE: Energy; HOM: Homogeneity; CON:

Contrast.

Table 3: Classification performance of the two machine learning classifiers with the selected features

\begin{tabular}{|c|c|c|c|c|c|c|}
\hline Classification Performance & Model & \%Sn & $\% \mathrm{~S}_{\mathrm{p}}$ & \%Acc & AUC & Best Feature(s) \\
\hline \multirow[t]{2}{*}{$\begin{array}{l}\text { First and second-order } \\
\text { (QUS+QUS-Tex }{ }^{1} \text { ) }\end{array}$} & $\mathrm{k}-\mathrm{NN}$ & 73 & 64 & 74 & 0.70 & $\begin{array}{l}\triangle \text { SAS } \\
\triangle \text { ASD-ENE } \\
\text { ASD-CON W0 }\end{array}$ \\
\hline & SVM & 74 & 86 & 84 & 0.78 & $\begin{array}{l}\text { SAS W0 } \\
\text { ASD-CON W0 } \\
\triangle \text { AAC-HOM }\end{array}$ \\
\hline \multirow[t]{2}{*}{$\begin{array}{l}\text { All features } \\
\text { (QUS+ QUS-Tex }^{1}+ \\
\text { QUS-Tex }^{1}-\text { Tex }^{2} \text { ) }\end{array}$} & k-NN & 87 & 75 & 81 & 0.83 & $\begin{array}{l}\text { ACE W0 } \\
\text { AAC-CON-CON wo } \\
\triangle \text { ASD-CON-CON }\end{array}$ \\
\hline & SVM & 75 & 85 & 85 & 0.78 & $\begin{array}{l}\text { SAS W0 } \\
\text { ASD-CON W0 } \\
\triangle \text { AAC-HOM }\end{array}$ \\
\hline
\end{tabular}

$* \Delta$ Indicates the difference of values of week 4 from week 0 for each feature included in the analysis

The best classifier performances using the k-NN model have been highlighted in bold.

Abbreviations: $\mathrm{S}_{\mathrm{n}}$ : Sensitivity; $\mathrm{S}_{\mathrm{p}}$ : Specificity, Acc: Accuracy, AUC: Area under curve; $k$ $\mathrm{NN}$ : $k$-nearest-neighbors; SVM: Support vector machine with radial based kernel function; AAC $\left(\mathrm{dB} / \mathrm{cm}^{3}\right)$ : Average Acoustic Concentration; $\mathrm{ASD}(\mu \mathrm{m})$ : Average Scatterer Diameter; SAS: Spacing Among Scatterer; ACE (dB/cm-MHz): Attenuation Coefficient Estimate; CON: Contrast; HOM: Homogeneity; ENE: Energy.

\section{Figures}


a
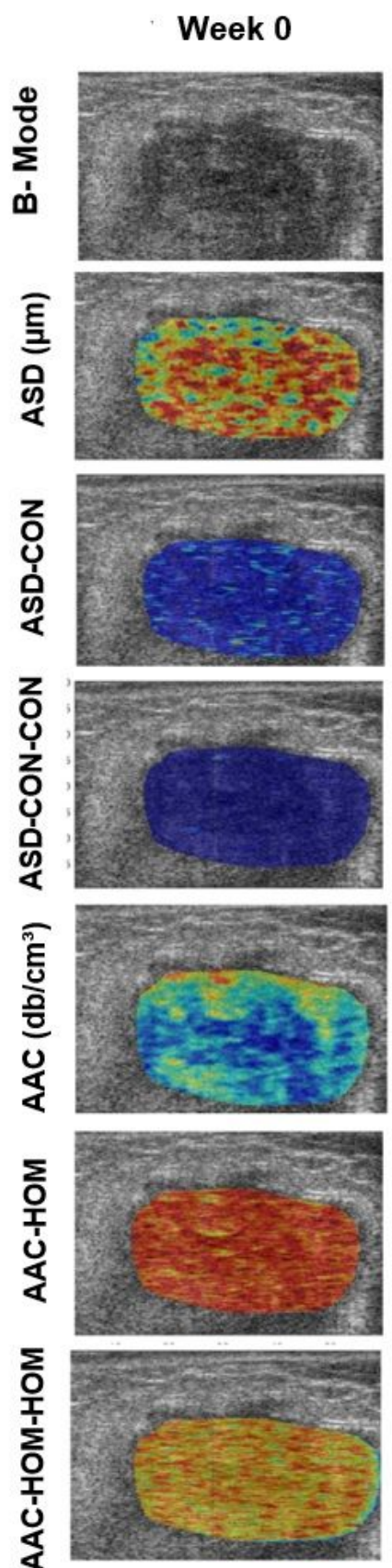

Week 4
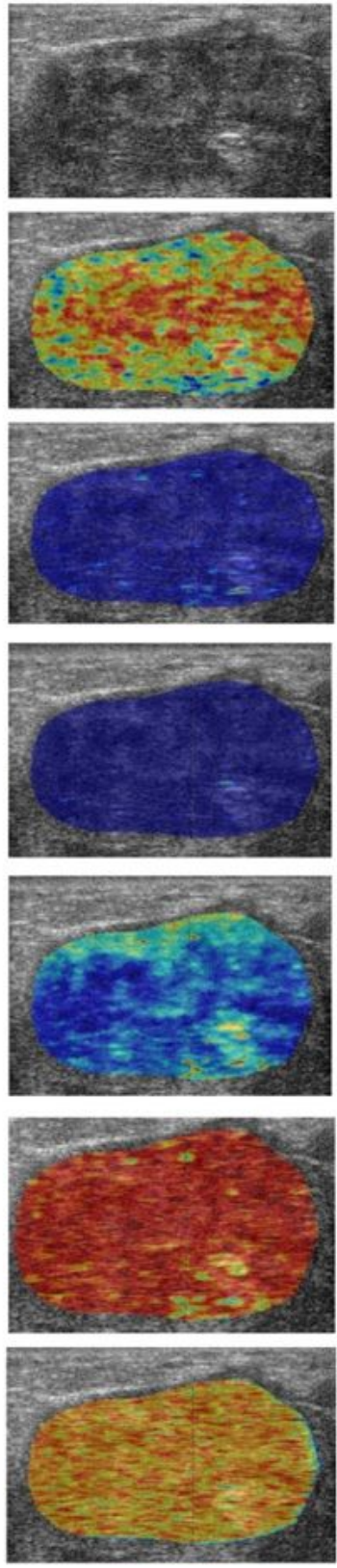

b Non-recurrence
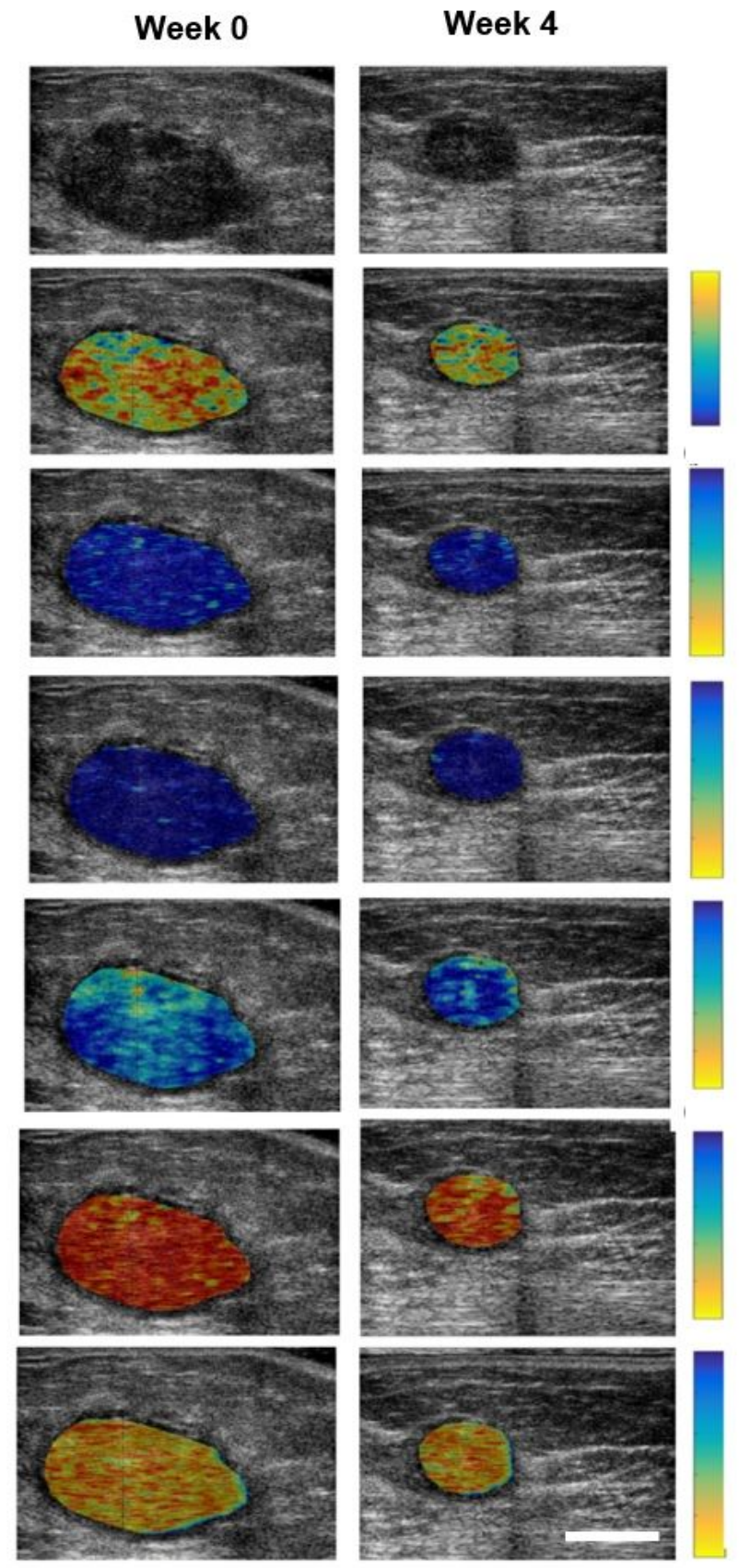
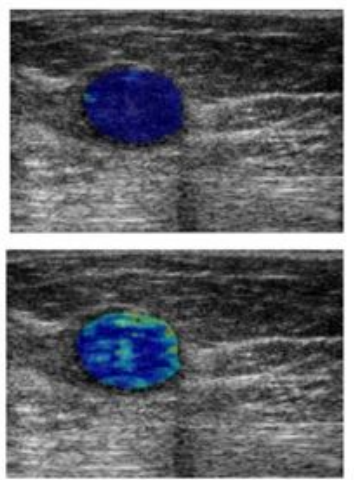

Week 4
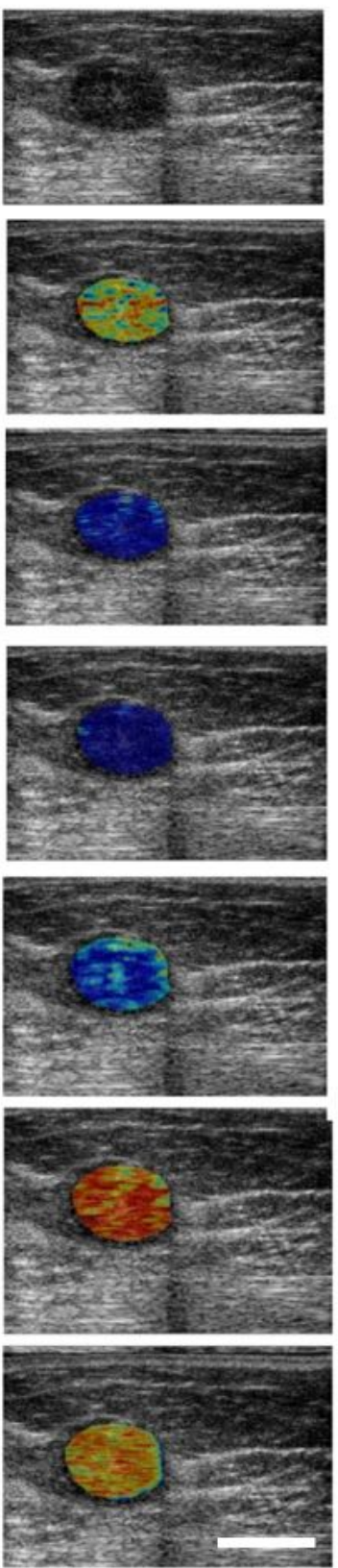

Figure 1

Quantitative ultrasound parametric images for representative patients with and without disease recurrence. Representative ultrasound B-mode images and QUS-derived parametric maps (ASD, ASD-CON, ASD-CON-CON, AAC, AAC-HOM, AAC-HOM-HOM) from one patient each with recurrence (left panel) and no recurrence (right panel) acquired at week 0 and week 4 of treatment. The color maps represent the quantitative values of the spectral parameters within the tumor. The change in values of the parameters with treatment can be appreciated by the change of assigned color to the sub-regions of interest within 
the tumor. The color scale on the right side represents the range for individual features, ASD parameter of 40 to $200 \mu \mathrm{m}, A S D-C O N$ texture feature of 0 to 20, ASD-CON-CON texture derivative of 0 to 54, AAC parameter of 7 to $65 \mathrm{db} / \mathrm{cm}^{3}, \mathrm{AAC}-\mathrm{HOM}$ texture feature of 0 to $1, \mathrm{AAC}-\mathrm{HOM}-\mathrm{HOM}$ texture derivative of 0 to 1. The scale bar represents $2 \mathrm{~cm}$.

a

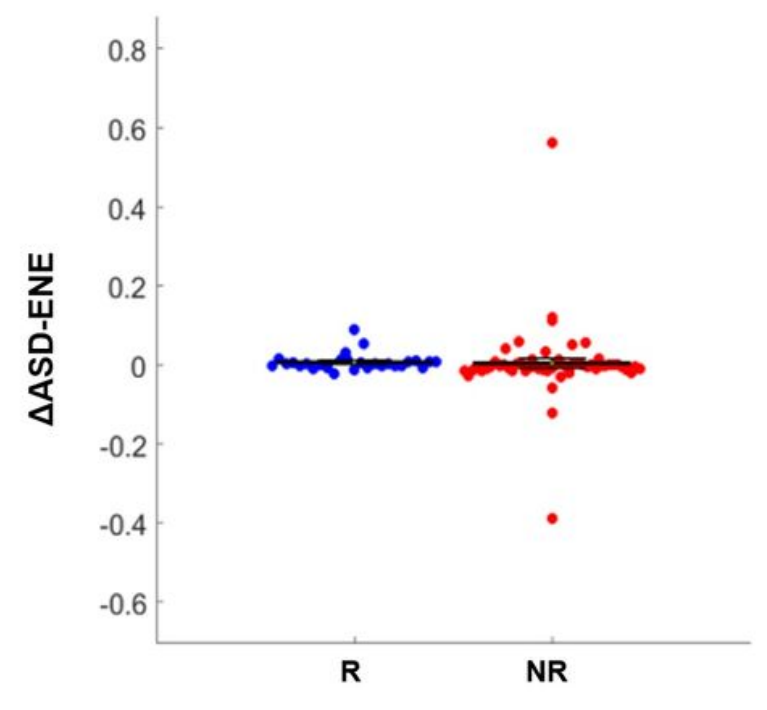

- Recurrence (R)

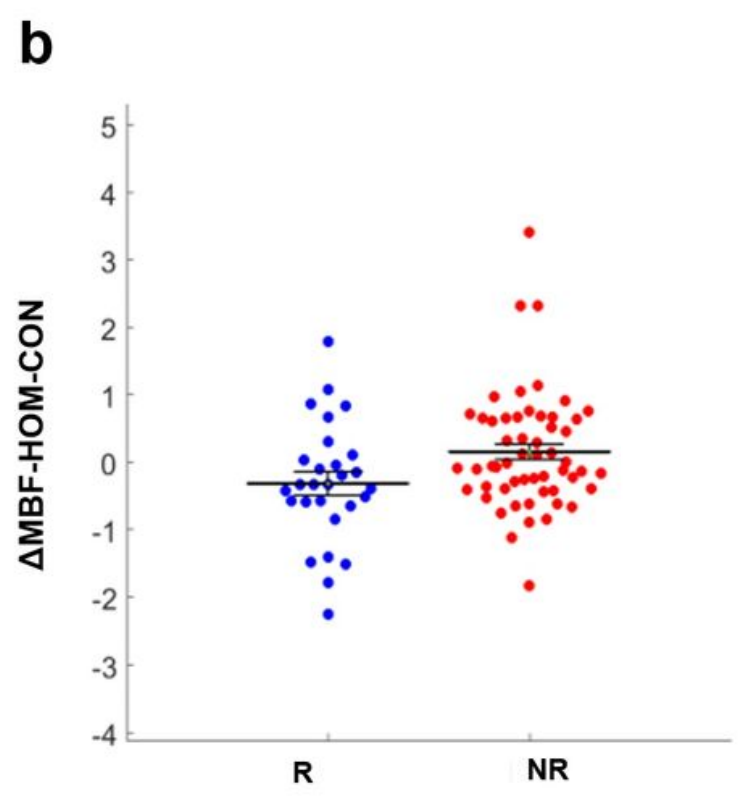

Non-recurrence (NR)

Figure 2

Scatter plots for the features having significantly different values for patients with and without recurrence. Figure 2 shows the distribution of values from all patients represented in the recurrence (blue circle) and without recurrence (red circle) groups for $\triangle$ ASD-ENE (2a) and $\triangle$ MBF-HOM-CON (2b). * $\Delta$ Indicates the difference of values of week 4 from week 0 for each feature were included in the analysis 


\section{k-NN}

\section{a}

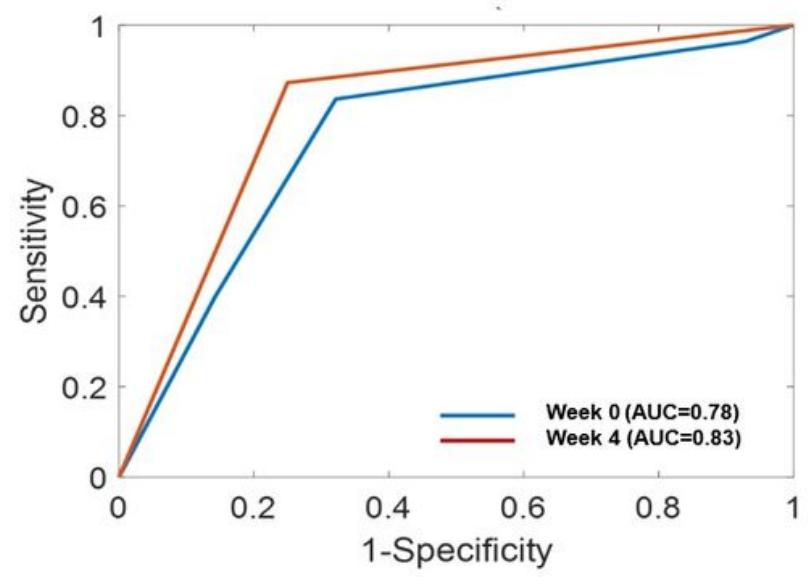

C

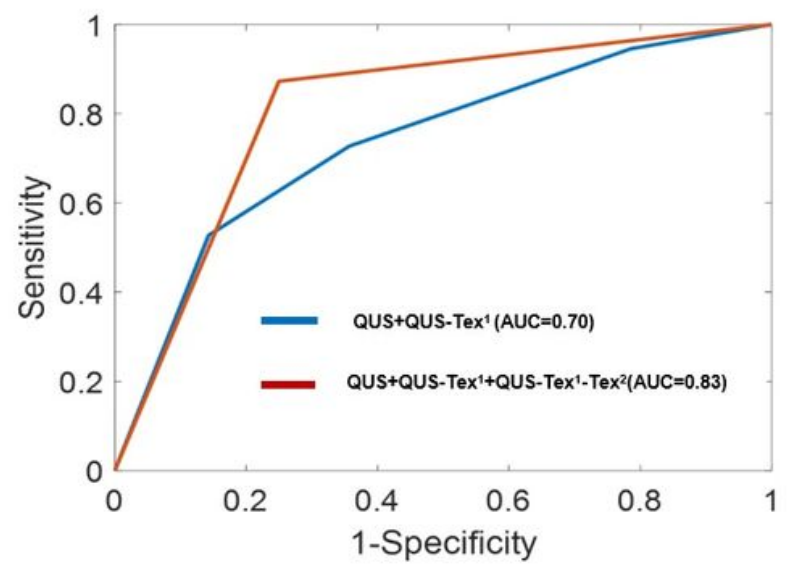

SVM

b
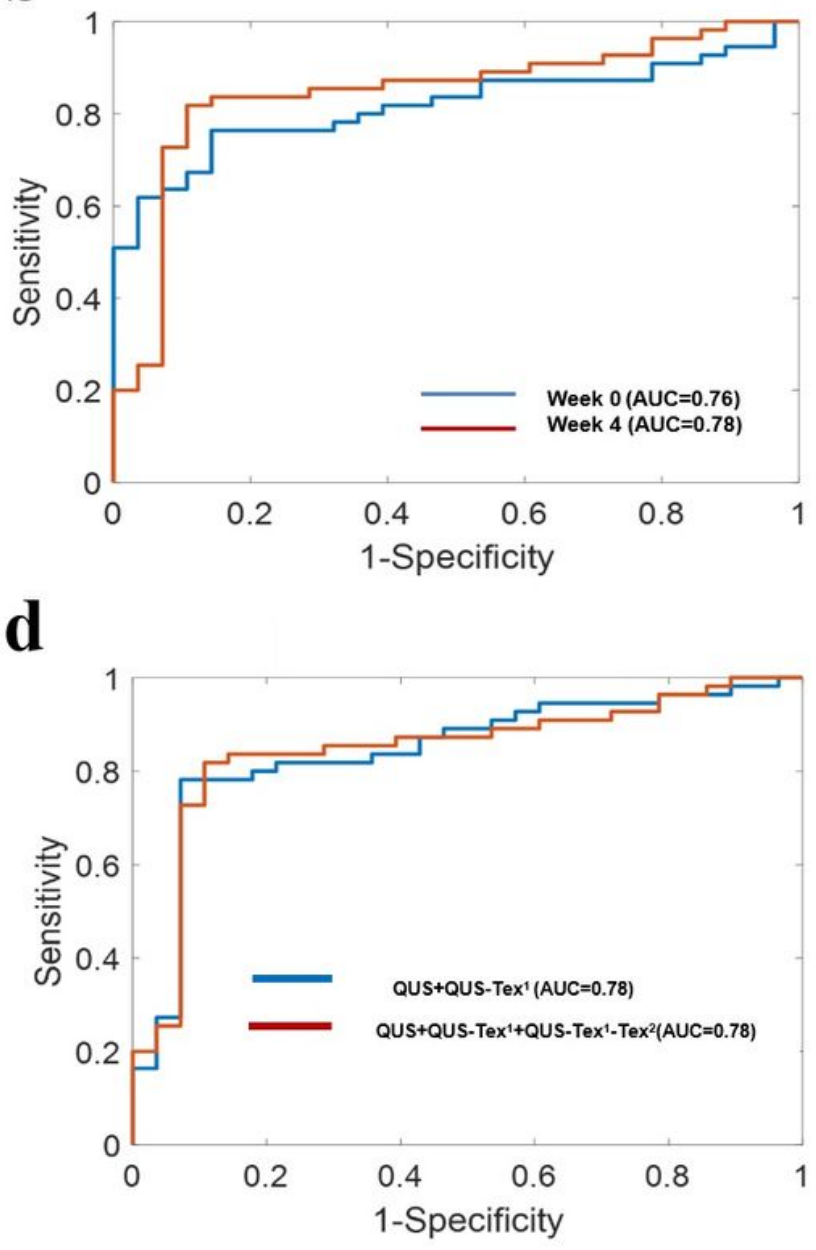

Figure 3

Receiver operating characteristic (ROC) plots showing the estimated area under curve (AUC) values obtained from the two classifiers. Figures $a \& b$ show a comparison of the performance by the two classifiers ( $k$-NN and SVM) based on week 0 and week 4 time-points using all features. Figures $\mathrm{c} \& \mathrm{~d}$ show the comparison of the performance of QUS + QUS-Tex1 ( without texture derivatives) and QUS + QUS-Tex1 + QUS-Tex1-Tex2 (with texture derivatives) data set by k-NN and SVM models at week 4. 
a

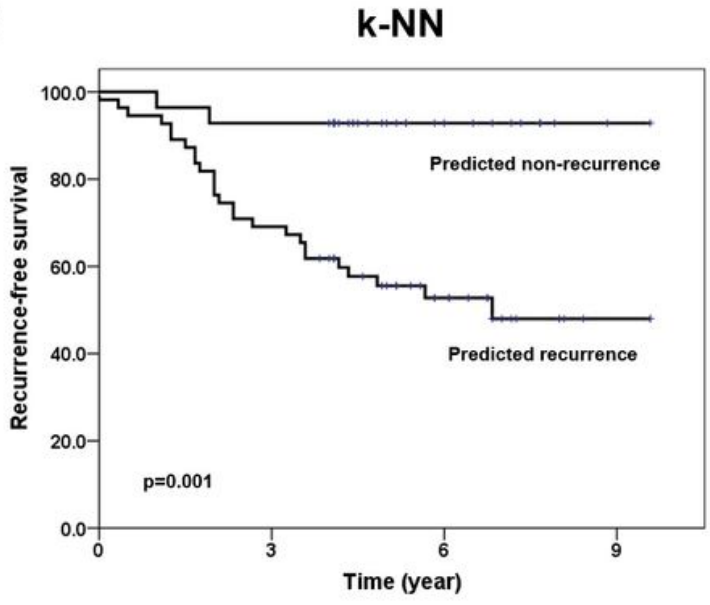

Numbers at risk

Predicted recurrence $\quad 28$ Predicted non-recurrence 55 b

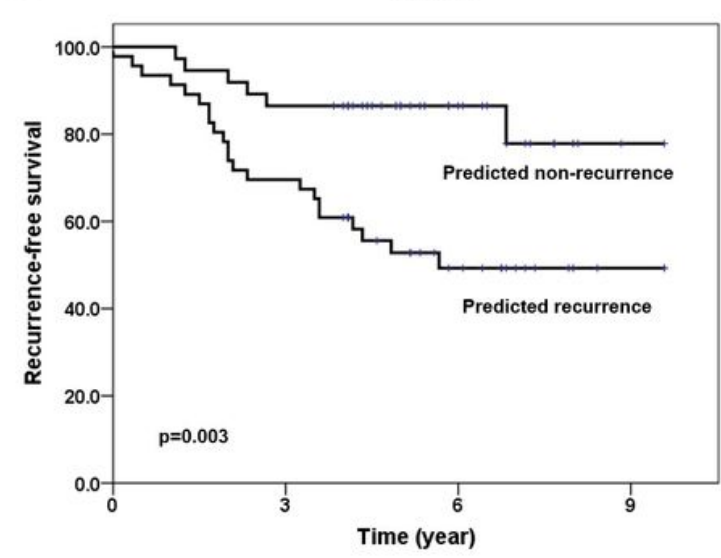

37
46

SVM

Figure 4

Kaplan Meier survival plots showing recurrence-free survival based on predicted groups (recurrence vs. no recurrence). Survival curves showing the differences in the recurrence-free survival outcomes as obtained from the predicted groups using the k-NN model (4a) and SVM model (4b) at week 4 time-point, including all features (QUS + QUS-Tex1 + QUS-Tex1-Tex2)).

\section{Supplementary Files}

This is a list of supplementary files associated with this preprint. Click to download.

- Supplementaryfigure1.pptx 